\title{
SIMULAÇÃO MONTE CARLO DE MECANISMO DE TRANSFERÊNCIA DE ENERGIA DE EXCITAÇÃO ELETRÔNICA: MODELO DE PERRIN PARA A SUPRESSÃO ESTÁTICA DA LUMINESCÊNCIA
}

\author{
João Batista Marques Novo e Lauro Camargo Dias Júnior*
}

Departamento de Química, Universidade Federal do Paraná, CP 19081, 81531-990 Curitiba - PR, Brasil

Recebido em 10/5/10; aceito em 21/10/10; publicado na web em 9/2/11

\begin{abstract}
MONTE CARLO SIMULATION OF ELECTRONIC EXCITATION ENERGY TRANSFER: PERRIN'S MODEL FOR STATIC LUMINESCENCE QUENCHING. A software based in the Monte Carlo method has been developed aiming the teaching of the Perrin's model for static luminescence quenching. This software allows the student to easily simulate the luminescence decays of emissive molecules in the presence of quenching ones. The software named PERRIN was written for FreeBASIC compiler and it can be applied for systems where the molecules remain static during its excited state lifetime. The good agreement found between the simulations and the expected theoretical results shows that it can be used for the luminescence and excited states decay kinetic teaching.
\end{abstract}

Keywords: Monte Carlo simulation; energy transfer; excited states decay kinetics.

\section{INTRODUÇÃO}

O entendimento dos processos envolvidos nos fenômenos de transferência de energia passa, muitas vezes, pela análise dos mecanismos cinéticos das populações dos estados eletrônicos excitados da amostra. Entender tais comportamentos cinéticos pode ser difícil e trabalhoso, pois muitas vezes estes processos apresentam equações matemáticas muito complexas. ${ }^{1}$ Neste sentido, o Método Monte Carlo (MC) é extremamente útil, pois permite a simulação de sistemas complexos de modo muito simples, sem que seja necessário saber, a priori, qual é a equação cinética associada. A denominação "Método Monte Carlo" tornou-se uma expressão geral associada ao uso de números aleatórios e de estatística de probabilidade. Para que a simulação $\mathrm{MC}$ esteja presente em um estudo, basta que este faça uso de números aleatórios na verificação do problema de interesse. ${ }^{2}$

Neste trabalho foi desenvolvido um software denominado "PERRIN" que faz simulações de supressão estática da luminescência molecular, para fins didáticos. O nome do software é devido a F. Perrin, que propôs, em 1924, um modelo para a supressão da emissão radiativa de moléculas eletronicamente excitadas $\left(\mathrm{M}^{*}\right)$ em meios rígidos. ${ }^{3,4} \mathrm{~A}$ supressão ocorre sempre que houver pelo menos uma molécula supressora (Q) dentro de uma esfera de volume $V_{\mathrm{q}}$ centrada na molécula emissora $\left(\mathrm{M}^{*}\right)$. As moléculas Q que estiverem situadas fora desta esfera - chamada por Perrin de "esfera ativa" - não irão desativar a emissão. Utilizamos o método de Monte Carlo, conforme o procedimento descrito mais adiante, para realizar o sorteio das moléculas a serem suprimidas. ${ }^{5}$

A probabilidade $\left(P_{\mathrm{m}}\right)$ de uma esfera ativa possuir um determinado número $(\mathrm{m})$ de moléculas supressoras é calculada pela distribuição de Poisson de acordo com: ${ }^{3,6}$

$$
P_{\mathrm{m}}=\left(<n>^{\mathrm{m}} / \mathrm{m} !\right) \exp (-<n>)
$$

onde $<n>$ é o número médio de moléculas supressoras dentro das esferas ativas, o qual é calculado através de:

*e-mail: laurocd@ufpr.br
$<n>=V_{\mathrm{q}} N_{\mathrm{a}}[Q]$

sendo $N_{\mathrm{a}}$ a constante de Avogadro e [Q] a concentração de supressor no meio, em mol $/ \mathrm{dm}^{3}$. A probabilidade da esfera não conter moléculas supressoras é obtida fazendo-se $\mathrm{m}=0$ resultando em:

$$
P_{0}=\exp (-<n>)
$$

Assim, tem-se que a intensidade de emissão luminescente $(I)$ de M será proporcional a $P_{0}$ de acordo com:

$$
I=I_{0} P_{0}=I_{0} \exp (-<n>)
$$

sendo $I_{0}$ a intensidade de emissão na ausência de supressor $([Q]=$ 0). Finalmente, substituindo-se a Equação 2 na Equação 4 chega-se à Equação de Perrin para a supressão estática da luminescência que é apresentada na seguinte forma:

$$
\left(I_{0} / I\right)=\exp \left(V_{\mathrm{q}} N_{\mathrm{a}}[Q]\right)
$$

ou ainda,

$$
\ln \left(I_{0} / I\right)=V_{\mathrm{q}} N_{\mathrm{a}}[Q]
$$

A distribuição de Poisson é um tipo de distribuição discreta de probabilidades sendo empregada em situações onde é possível a ocorrência de um grande número de eventos, porém, com cada um deles ocorrendo com baixa probabilidade. ${ }^{6}$ Este tipo de distribuição é bastante comum em espectroscopia de luminescência resolvida no tempo, pois os dados obtidos por meio da técnica de contagem de fótons únicos (Single Photon Counting) exibem estatística de contagem nuclear. ${ }^{7}$ Também é utilizada na determinação do número de agregação micelar através de técnicas de supressão estática de luminescência - neste caso pode-se prever a probabilidade de uma determinada micela possuir uma ou mais moléculas supressoras no seu interior. ${ }^{3}$ 
Um gráfico de $I_{0} / I$ versus $[Q]$ mostra que a Equação de Perrin não é linear. Este modelo difere daquele de Stern-Volmer, que é aplicado para processos de supressão dinâmica, ou seja, dependentes da colisão entre $\mathrm{M}$ e Q. Neste caso, há uma resposta linear entre $I_{0} / I$ e $[Q]$, conforme a Equação de Stern-Volmer (7): ${ }^{3,4}$

$$
\left(I_{0} / I\right)=1+k \tau[Q]
$$

onde $k$ é a constante de velocidade para o processo (podendo-se ter $k \approx k_{\text {difusional }}$ ) e $\tau$ o tempo de vida de $\mathrm{M}^{*}$. Assim, é possível diferenciar qual o tipo de supressão operante (estática ou dinâmica) e ainda se há a presença de ambos os processos.

O modelo de Perrin descreve bem os processos de supressão estática de curto alcance, pois os valores experimentais de $V_{\mathrm{q}} N_{\mathrm{a}} \cos -$ tumam situar-se entre 1 e $3 \mathrm{dm}^{3} / \mathrm{mol}$, resultando em raios da ordem de alguns Angstroms. O raio $(R)$ da esfera ativa em Angstroms é calculado através de:

$$
R=\left[\left(3 \times 10^{24} V_{\mathrm{q}}\right) /(4 \pi)\right]^{1 / 3}
$$

Como exemplo, mecanismos de interação de troca de elétrons fornecem raios entre 10 e $15 \AA$ A. Também são encontrados na literatura refinamentos do modelo que levam em consideração um volume excluído (correspondente aos volumes das moléculas M e Q) como no estudo de transferência de energia em matrizes poliméricas. ${ }^{8-10}$

\section{O PROGRAMA DESENVOLVIDO E O MÉTODO DE SORTEIO}

O programa de nome PERRIN, implementado neste trabalho, foi disponibilizado para uso aos interessados. ${ }^{11}$ Foi utilizado o FreeBASIC, um compilador BASIC de código aberto e gratuito. ${ }^{12,13} \mathrm{~A}$ entrada de dados consiste no dimensionamento dos seguintes parâmetros: a) da aresta $(A)$ de um "universo cúbico"; b) do número de moléculas luminescentes $\left(N_{M}\right)$ e supressoras $\left(N_{Q}\right)$; c) do raio $(R)$ da esfera de supressão (esfera ativa) centrada em cada molécula luminescente e, d) do número de jogadas (sorteios) do método MC.

Para que a simulação apresente resultados compatíveis com os obtidos experimentalmente, tanto a aresta do "universo cúbico" $(A)$ como o raio $(R)$ da "esfera ativa" devem ser definidos na escala de Angstroms. Uma boa tentativa é considerar $A \approx R N_{M}{ }^{1 / 3}=10 N_{M}^{1 / 3}$, que seria válido para moléculas luminescentes onde $R=10 \AA$.

No software, as concentrações de moléculas supressoras são calculadas na escala microscópica, que é dada pela expressão:

$$
[\mathrm{Q}]_{\mathrm{mi}}=N_{u} / V_{u}
$$

onde $N_{u}$ é o número de moléculas no universo cúbico e $V_{u}$ é o seu volume em $\AA^{3}$. Na escala macroscópica, o valor correspondente, em $\mathrm{mol} / \mathrm{dm}^{3}$, é:

$$
[\mathrm{Q}]=[\mathrm{Q}]_{\mathrm{mi}} \times 10^{27} / N_{A}=1660,54 N_{u} / V_{u}
$$

onde $N_{A}=6,02214 \times 10^{23} \mathrm{~mol}^{-1}$ é a Constante de Avogadro e $10^{27}$ é o fator de conversão entre $\mathrm{dm}^{3}$ e $\AA^{3}$. Tem-se, portanto, que para se obter a concentração em quantidade de matéria $\left(\mathrm{mol} / \mathrm{dm}^{3}\right)$ basta multiplicar a concentração de moléculas a nível microscópico, pela constante 1660,54.

O método de sorteio emprega o modelo de urna de Ehrenfest e o programa principal consiste nas seguintes etapas: ${ }^{5} 1$ ) sortear as posições (coordenadas) das moléculas luminescentes (M) e supressoras (Q) no universo; 2) sortear uma molécula $\mathrm{Me}$, se ela existir no estado excitado $\left(\mathrm{M}^{*}\right)$, prossegue-se para o próximo item; 3) calculam-se as distâncias até as moléculas Q; 4) se houver alguma molécula Q cuja distância a $\mathrm{M}^{*}$ for menor que $R$, haverá supressão da luminescência (não emite fóton); 5) caso contrário, $\mathrm{M}^{*}$ emitirá um fóton; 6) contase o número de fótons emitidos a cada 100 sorteios; 7) o gráfico do decaimento de luminescência (número de fótons emitidos em função do número de jogadas), para um determinado número de moléculas supressoras $\left(N_{Q}\right)$ é mostrado na tela; 8$)$ após todas as simulações, com diferentes valores de $N_{Q}$, o gráfico de Perrin, $\ln \left(N_{0} / N\right)$ versus $[Q]$ é mostrado na tela.

\section{RESULTADOS E DISCUSSÃO}

As simulações foram realizadas com $A=100, N_{M}=10000, N_{Q}$ $=100,200,400,800,1600,3200$ e $6400, R=3,0 ; 3,5 ; 4,0$ e 4,5 e número de jogadas $=30000$.

A Figura 1 apresenta as curvas de decaimento dos estados excitados das moléculas luminescentes (gráfico do número de fótons emitidos em função do número de jogadas) na presença de 800 , 3200 e 6400 moléculas supressoras. Pode-se notar neste gráfico que o aumento do número de moléculas supressoras diminui o número de fótons emitidos num determinado tempo (jogada), ou seja, ocorre supressão da energia de excitação eletrônica.

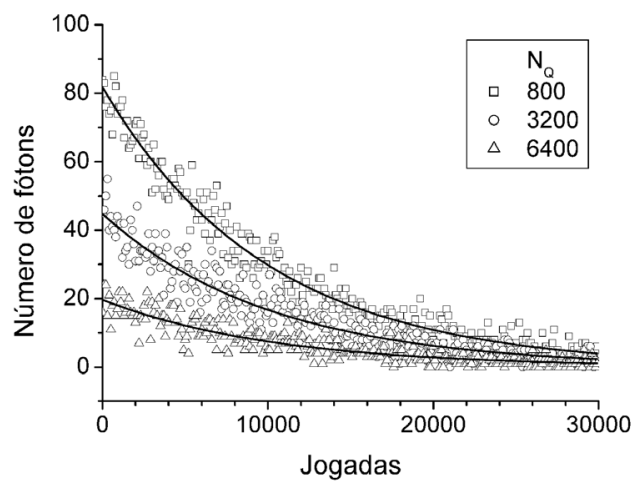

Figura 1. Simulações Monte Carlo das curvas de decaimento de estado excitado obtidas a partir de $A=100, N_{M}=10000, R=4$ e número de jogadas = 30000. As curvas contínuas foram ajustadas por regressão não linear aos pontos simulados. Para clareza, são mostradas nesta figura apenas as simulações obtidas a partir de $N_{Q}=800,3200$ e 6400 moléculas supressoras

Outra característica importante destas curvas de decaimento é que elas têm formato exponencial, que pode ser evidenciado pelo gráfico semilogarítmico mostrado na Figura 2. Assim, as três curvas apresentadas possuem o mesmo coeficiente angular (a mesma inclinação), que é característico de decaimento de primeira ordem. Isto indica que M mantém o mesmo tempo de vida de estado excitado, independentemente do número de moléculas supressoras presentes no sistema. Este comportamento, característico do mecanismo de supressão de energia de Perrin, difere daquele de Stern-Volmer, onde o tempo de vida de estado excitado diminui com o aumento de $[Q]$. $^{3,4}$

O número máximo de jogadas escolhido na simulação é aquele necessário para se obter uma curva de decaimento exponencial, na sua totalidade. Por tentativa e erro, foi verificado que esta condição pode ser obtida quando este número é igual a $3 N_{M}$ sendo que o software fornece, de forma padrão, o número de jogadas adequado.

Verificou-se também que a dispersão dos pontos simulados com relação à curva de decaimento exponencial é grande. Isto é devido ao fato de que o número de fótons emitidos é contado em intervalos de 100 jogadas. O usuário pode, entretanto, aumentar este valor para 1000 (através da variável NJogFotonsINT) de modo a diminuir esta dispersão. 


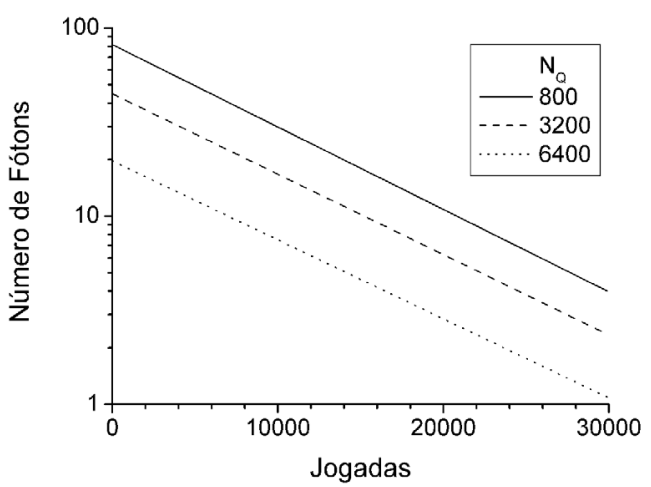

Figura 2. Gráfico semilogarítmico das curvas ajustadas por regressão não linear, presentes na Figura 1. São mostradas as curvas obtidas com $N_{Q}=800$, 3200 e 6400 moléculas supressoras

A Figura 3 apresenta as simulações correspondentes à Equação de Perrin (Equação 6). Para cada curva de decaimento simulada, $N_{0}$ e $N$ correspondem ao número de fótons emitidos no tempo inicial (jogada $=0$ ), na ausência e na presença de moléculas supressoras, respectivamente; $[Q]_{\mathrm{mi}}$ é a concentração de moléculas supressoras, calculada dividindo-se $N_{Q}$ pelo volume do universo (aresta do universo elevada ao cubo, $A^{3}$ ). Para se construir esta Figura foram necessárias as simulações de curvas de decaimentos com diferentes quantidades de moléculas supressoras $\left(N_{Q}\right)$ e, também, com diferentes raios da esfera de supressão $(R)$ das moléculas doadoras.

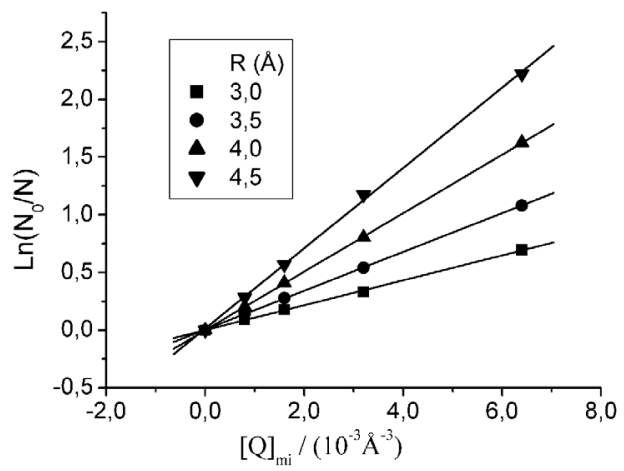

Figura 3. Gráficos de Perrin para as simulações Monte Carlo. São mostrados os pontos obtidos a partir de simulações com diferentes concentrações de moléculas supressoras $\left([Q]_{m i}=N_{\complement} / A^{3}\right)$ e com diferentes raios da esfera de supressão das moléculas doadoras (R). Para clareza, são mostrados apenas os pontos obtidos com $N_{Q}=0,800,1600,3200$ e 6400 moléculas supressoras. Mais informação no texto

Observa-se que os gráficos da Figura 3 são lineares para cada valor de $R$, e que os coeficientes angulares (inclinação) destas retas aumentam com o valor de $R$, conforme previsto pelas Equações 6 e 8. Isto é evidenciado na Figura 4, que apresenta o gráfico destes coeficientes angulares versus $R^{3}$. Observa-se que a dependência das inclinações dos gráficos de Perrin é função linear do volume da esfera ativa $\left(R^{3}\right)$, pois um aumento no volume da esfera ativa aumenta a probabilidade de se encontrarem moléculas supressoras dentro desta.

Cabe ressaltar que não é possível simular o modelo de SternVolmer no software atual, pois o algoritmo implementado trata da supressão estática de energia de excitação. Já o modelo de SternVolmer (Equação 7) não apresenta a limitação do raio da esfera ativa e haveria sempre a competição entre dois canais de desativação

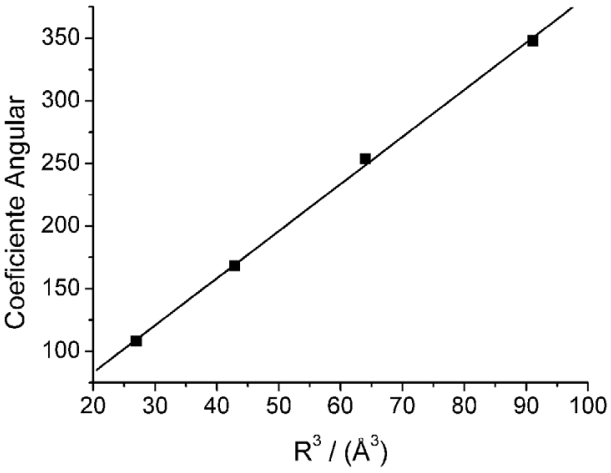

Figura 4. Coeficientes angulares dos Gráficos de Perrin da Figura 3 em função de $R^{3}$

dos estados excitados, dependendo da distância entre as moléculas emissoras e supressoras e, também, da taxa de colisões entre estas moléculas (supressão dinâmica). ${ }^{3,4} \mathrm{~A}$ implementação do modelo de Stern-Volmer requer um novo algoritmo, que estamos considerando como perspectiva de trabalho futuro.

\section{CONSIDERAÇÕES FINAIS}

Neste trabalho, foram apresentadas várias simulações Monte Carlo dos decaimentos de estado excitado de moléculas luminescentes na presença de moléculas supressoras, de acordo com o mecanismo de supressão de energia eletrônica de Perrin. Os excelentes resultados obtidos evidenciam que o software desenvolvido está funcionando adequadamente e que pode ser utilizado no ensino de mecanismos de luminescência e de cinética de estados excitados. O Método Monte Carlo permite ao aluno facilmente simular e visualizar os mecanismos de luminescência, sem a necessidade de se conhecer as equações analíticas associadas e o programa de simulação PERRIN está disponível para uso. ${ }^{11}$

\section{REFERÊNCIAS}

1. Birks, J. B.; Photophysics of Aromatic Molecules, Wiley-Interscience: London, 1970.

2. Angelotti, W. F. D.; Fonseca, A. L.; Torres, G. B.; Custódio, R.; Quim. Nova 2008, 31, 433.

3. Valeur, B.; Molecular Fluorescence, Principles and Applications, WileyVCH: Weinheim, 2001.

4. Turro, N. J.; Modern Molecular Photochemistry, University Science Books: Sausalito, 1991.

5. López-Castillo, A.; Souza-Filho, J. C.; Quim. Nova 2007, 30, 1759.

6. http://mathworld.wolfram.com, acessada em Setembro 2010 e Janeiro 2011.

7. Demas, J. N.; Excited State Lifetime Measurements, Academic Press: New York, 1983.

8. Merkel, P. B.; Dinnocenzo, J. P.; J. Phys. Chem. A 2008, 112, 10790.

9. Yang, J.; Winnik, M. A.; Can. J. Chem. 1995, 73, 1823.

10. Daglen, B. C.; Tyler, D. R.; Macromolecules 2008, 41, 9525.

11. http://www.quimica.ufpr.br/jbmnovo/perrin, acessada em Maio 2010 e Janeiro 2011.

12. Victor, A.; FreeBASIC; A Free BASIC Compiler; http://www.freebasic. net, acessada em Maio 2010 e Janeiro 2011.

13. Debord, J.; FBMath; FreeBasic Math Library; http://sourceforge.net/ projects/fbmath, acessada em Maio 2010 e Janeiro 2011. 